\title{
Examining the Effect of Pileup on the Accuracy of Sharp Indentation Testing
}

\author{
Zhongbin Tang, ${ }^{1}$ Yazhou Guo, ${ }^{1}$ Zhaohu Jia, $^{2}$ Yulong Li, ${ }^{1}$ and Qiuming Wei ${ }^{3}$ \\ ${ }^{1}$ School of Aeronautics, Northwestern Polytechnical University, Xi'an 710072, China \\ ${ }^{2}$ Xian ASN Technology Group Co. Ltd., Northwestern Polytechnical University, Xian 710072, China \\ ${ }^{3}$ Department of Mechanical Engineering, University of North Carolina at Charlotte, Charlotte, NC 28277, USA
}

Correspondence should be addressed to Yazhou Guo; guoyazhou@nwpu.edu.cn

Received 9 January 2015; Revised 26 May 2015; Accepted 27 May 2015

Academic Editor: Francesco Delogu

Copyright (C) 2015 Zhongbin Tang et al. This is an open access article distributed under the Creative Commons Attribution License, which permits unrestricted use, distribution, and reproduction in any medium, provided the original work is properly cited.

\begin{abstract}
One essential problem related to instrumented indentation is the effect of pileup, which could introduce significant errors on the measured hardness and elastic modulus. In this work, we have assessed some critical issues associated with instrumented indentation by means of numerical simulation. Dimensional analysis is adopted to acquire the principal governing parameters of the process, such as the ratio of yield strength to elastic modulus $(Y / E)$ and the ratio of indentation depth to maximum penetration depth $\left(h / h_{\max }\right)$. The dimensional analysis provides a general understanding on the relationships among these dimensionless variables and their influence on the test results. Three kinds of indenters, that is, conical, Berkovich, and Vickers indenter, are comparatively studied. The dependence of pileup on the dimensionless parameters and its effect on the simulation results are also given. These findings are instructive for measuring the mechanical properties by instrumented indentation, especially when the effect of pileup is involved.
\end{abstract}

\section{Introduction}

Instrumented indentation, particularly nanoindentation, has been widely used for characterizing the mechanical properties of materials at small scales [1-6]. One of the great advantages of this technique is that the mechanical properties, such as hardness and elastic modulus, can be directly determined from the indentation load-displacement curves, while the measurement of the actual hardness impression is not necessary. Recent developments of the nanoindentation technique make it capable of recording experimental data at load and displacement as small as micro-Newton and nanometer levels.

The commonly used definition of hardness is as follows:

$$
H=\frac{P}{A},
$$

where $H$ is hardness, $P$ the load applied to the test surface, and $A$ the projected contact area. $A$ was calculated by some researchers using the following equation $[1,4]$ :

$$
A=f_{1}\left(h_{c}\right)=f_{2}(h),
$$

where $h_{c}$ is the contact depth and $h$ is the resulting penetration depth. If pileup effect is negligible, $h_{c}$ could be given by

$$
h_{c}=h-\frac{\varepsilon P}{S},
$$

where $\varepsilon$ is a constant that depends on indenter geometry and $S$ is the elastic stiffness of the contact [4]. One should notice that (3) cannot account for the plastic phenomenon of pileup, which may lead to significant change of the true contact area.

The elastic unloading curve of nanoindentation is approximately expressed in the power-law relation $[4,7]$ :

$$
\frac{P}{P_{\max }}=\alpha\left(\frac{h-h_{f}}{h_{\max }-h_{f}}\right)^{n}
$$

where $\alpha$ and $n$ are empirically determined fitting parameters, depending on the geometry of the indenter. $h_{f}$ is the final displacement of the indenter after complete unloading. Then 
$S$ could be acquired by calculating the slope of the initial portion of the unloading curve:

$$
S=\left.\frac{d P}{d h}\right|_{h=h_{\max }}=\alpha P_{\max } n\left(h_{\max }-h_{f}\right)^{-1} .
$$

The relationship between the effective elastic modulus and the projected contact area and unloading stiffness is given by $[1$, 4]

$$
E_{\text {eff }}=\frac{\sqrt{\pi} \times S}{2 \beta \sqrt{A}},
$$

where $\beta$ is a dimensionless factor that accounts for deviations in stiffness caused by the lack of axial symmetry for Berkovich or Vickers indenters. The effective elastic modulus is defined by

$$
\frac{1}{E_{\mathrm{eff}}}=\frac{1-v^{2}}{E}+\frac{1-v_{i}^{2}}{E_{i}}
$$

where $E$ and $v$ are elastic modulus and Poisson's ratio of the specimen and $E_{i}$ and $v_{i}$ are those of the indenter. The elastic modulus of the specimen, $E$, could then be calculated by (7). In this work, the indenter is taken as rigid body for numerical simulation, and then $E=E_{\text {eff }}\left(1-v^{2}\right)$.

From (1) to (7), the hardness as well as the elastic modulus of the material could be determined in the following sequence:

(a) Fit the unloading curve of the indentation; find values of $\beta$ and $n$, and determine $S$ by (5).

(b) Find contact depth $h_{c}$ by (3).

(c) Determine the projected contact area $A$ by (2).

(d) Obtain hardness by (1).

(e) Derive $E_{\text {eff }}$ by (6) and then elastic modulus $E$ by (7).

The difficulty related to the above method is the determination of the contact depth $h_{c}$ and the true projected contact area $A$, especially when the phenomenon of pileup occurs. The resulting contact area might be greatly underestimated by using these equations if pileup effect exists. Bolshakov and Pharr [7] found by numerical simulation that pileup was significant for conical indenter if the ratio of final displacement to the maximum depth of penetration is larger than 0.7 (i.e., $h_{f} / h_{\max }>0.7$ ). Y.-T Cheng and C.-M Cheng $[2,8-11]$ did a series of analysis on conical indentation by finite element method (FEM). They analyzed their data by the method of dimensional analysis, which provided us a general understanding of this process. The dependencies of $H$ and $E$ on some critical dimensionless variables, such as $Y / E, \nu$, and $\theta(Y$ is the yield strength of the tested material and $\theta$ is the half angle of the indenter), were investigated. However, their numerical simulations were limited to $2 \mathrm{D}$, which may represent the conical indentation fairly well but are not so representative for some 3D indenters such as Berkovich indenter and Vickers indenter. Dao et al. [12] conducted comprehensive analysis on the data derived from indentation by finite element simulation, where they also used 2D axial symmetric model instead of the real configuration. Giannakopoulos et al. [13] and Larsson et al. [14] investigated Vickers indenter and Berkovich indenter separately, but detailed comparison among these sharp indenters, such as Vickers, Berkovich, and conical, was not provided. Although the geometry functions of Berkovich indenter and Vickers indenter can be designed to be the same as that of the conical indenter, the true projected contact areas at the same indentation depth may still be different considering the effect of pileup.

Apart from geometric difference of indenters, there are several other factors that may influence the measurement of instrumented indentation. The thickness and properties of the test materials and the substrate effect have been studied systematically by many researchers [16-21]. The stress state within the material also affects the testing results of indentation [22, 23]. In fact, Larsson et al. successfully related stress state to indentation testing results and found a way of determining residual stress within the material $[24,25]$ by sharp indentation.

In this paper, we will focus on the differences of indenter geometries and their effect on the indentation results by means of numerical simulation. Dimensional analysis will be adopted to acquire the principal governing parameters. The effect of pileup on the simulation results will also be given.

Three kinds of indenters, namely, conical indenter, Berkovich indenter, and Vickers indenter, will be discussed separately. The half angle of the conical indenter is set to be $70.3^{\circ}$, while the half angles of Berkovich indenter and Vickers indenter are chosen to be $65.3^{\circ}$ and $68^{\circ}$, respectively. The relationship between the projected contact area $A$ and the contact depth $h_{c}$ (i.e., $f_{1}\left(h_{c}\right)$ ) can be obtained through simple calculation and all of the three indenters have equivalent geometry functions:

$$
A=f_{1}\left(h_{c}\right) \approx 24.52 h_{c}^{2} \text {. }
$$

\section{Dimensional Analysis}

2.1. Dimensional Analysis for Loading. For a simple instrumented indentation test, the procedure is composed of two steps, that is, loading and unloading. During the loading process, the produced load $P$ and projected contact area $A$ are functions of all the independent governing parameters, which include the material parameters (Young's modulus $E$, yield strength $Y$, and Poisson's ratio $v$ ), the indenter geometry parameter (those in (2)), and the indentation depth $h$. For a given indenter, $P$ and $A$ can be written as

$$
\begin{aligned}
& P=f_{p L}(E, Y, v, h), \\
& A=f_{A}(E, Y, v, h) .
\end{aligned}
$$


Among these governing parameters, we may choose two basic dimensions, that is, $[E]$ and $[h]$. The dimensions of the rest of the variables can then be described as

$$
\begin{aligned}
& {[P]=[E][h]^{2},} \\
& {[A]=[h]^{2},} \\
& {[Y]=[E],} \\
& {[\nu]=[E]^{0}[h]^{0} .}
\end{aligned}
$$

Since the influence of $v$ on the measurement of hardness and elastic modulus is relatively small [1] (e.g., $v=0.25 \pm 0.1$ produces only about $5 \%$ uncertainty in the calculated value of $E$ ), we will not incorporate the effect of $\nu$ for simplicity in this work. Conducting dimensional analysis by applying the $\Pi$ theorem [26], the following equations can be obtained:

$$
\begin{aligned}
& \psi_{\Pi}\left(\Pi_{p 1}, \Pi_{p 2}\right)=0, \\
& \varphi_{\Pi}\left(\Pi_{A 1}, \Pi_{A 2}\right)=0,
\end{aligned}
$$

where $\Pi_{P 1}=P E^{-1} h^{-2}, \Pi_{P 2}=\Pi_{A 2}=Y E^{-1}$, and $\Pi_{A 1}=A h^{-2}$. Then the above equations can be rewritten as

$$
\begin{aligned}
& P=E h^{2} \phi_{P L}\left(\frac{Y}{E}\right), \\
& A=h^{2} \phi_{A}\left(\frac{Y}{E}\right) .
\end{aligned}
$$

Note that $\phi_{P L}$ and $\phi_{A}$ are different functions from $\psi_{\Pi}$ and $\varphi_{\Pi}$, respectively. It is easy to see from (12) and (13) that $P$ and $A$ are all proportional to $h^{2}$. The hardness can then be calculated by

$$
H=\frac{P_{\max }}{A}=E \phi_{H}\left(\frac{Y}{E}\right) .
$$

Clearly, the hardness depends only on the material parameters $E$ and $Y$, and it is independent from the indentation depth. Moreover, the dimensionless hardness, that is, $H / E$, is a function of only one parameter $Y / E$ from (14).

2.2. Dimensional Analysis for Unloading. During unloading, the imposed load $P$ depends on the following independent variables: $E, Y, h$, and maximum indentation depth $h_{\max }$, while the final indentation depth $h_{f}$ depends only on $E, Y$, and $h_{\max }$ (the effect of $v$ is not considered here). We have

$$
\begin{aligned}
P & =f_{P U}\left(E, Y, h, h_{\max }\right), \\
h_{f} & =f_{h f}\left(E, Y, h_{\max }\right) .
\end{aligned}
$$

Again, the dimensional analysis gives

$$
\begin{aligned}
P & =E h^{2} \phi_{P U}\left(\frac{Y}{E}, \frac{h}{h_{\max }}\right), \\
h_{f} & =h_{\max } \phi_{h f}\left(\frac{Y}{E}\right) .
\end{aligned}
$$

Equation (17) indicates that the final indentation depth is proportional to the maximum indentation depth and is dependent on $Y / E$. Since the slope of the unloading curve is essential for calculating $E$ and $H$, we take derivative with respect to $h$ on both sides of (16) and obtain the values at $h=h_{\max }$ :

$$
\left.\frac{d P}{d h}\right|_{h=h_{\max }}=E h_{\max }\left[2 \phi_{P U}\left(\frac{Y}{E}, 1\right)+\dot{\phi_{P U}}\left(\frac{Y}{E}, 1\right)\right] .
$$

Or equivalently,

$$
\begin{aligned}
\left.\frac{d P}{d h}\right|_{h=h_{\max }} & =S=\left[2 \phi_{P U}\left(\frac{Y}{E}, 1\right)+\dot{\phi_{P U}}\left(\frac{Y}{E}, 1\right)\right] \\
& =E h_{\max } \phi_{S}\left(\frac{Y}{E}\right) .
\end{aligned}
$$

The dimensionless form of the elastic contact stiffness $S /\left(E h_{\max }\right)$ depends on $Y / E$.

\section{Numerical Analysis}

Finite element analysis (FEA) of the indentation process has been carried out by using the commercial software Abaqus. The above-mentioned scaling relationships are carefully examined. These dimensionless functions, such as $\phi_{P L}$, $\phi_{A}, \phi_{h f}$, and $\phi_{S}$, are evaluated. The effect of pileup on the calculated hardness and elastic modulus will also be discussed.

As mentioned before, three kinds of indention, namely, conical, Vickers, and Berkovich, are simulated. All of the indenters have the same projected area function (8). The dimension of the tested specimen was set to be larger than ten times of the contact length in every direction to minimize the influence of the boundary. Similar to experimental test, the bottom surface of the specimen is set to be fixed and all of the remaining surfaces are set to be free. The indenter is free along the indentation direction only and the loads are given through applying displacements on the indenter. The contact between the indenter and specimen is set to be frictionless and the whole process is considered to be time-independent.

The indenters were taken as rigid body, while the tested materials were regarded as elastic-perfect-plastic. The yield strengths of the materials are set to range from $80 \mathrm{MPa}$ to $8 \mathrm{GPa}$, which covers most of the engineering materials. Two elastic modulus values, that is, $100 \mathrm{GPa}$ and $200 \mathrm{GPa}$, are considered. Poisson's ratio is set to be 0.38 , corresponding to a material of niobium that will be used for comparison with the experimental observations. Actually, the values of Poisson's ratio taken from 0.2 to 0.4 will not lead to any significant difference [8].

A total number of 60,000 brick elements are meshed in the material and about 3,000 quadrilateral elements are meshed in the indenter. An example of the mesh of half of the conical indentation model is given in Figure 1. Intense mesh is given in the region of contact and sparse mesh in the far field to compromise between accuracy and efficiency. Mesh dependence of the simulation results was conducted first and 


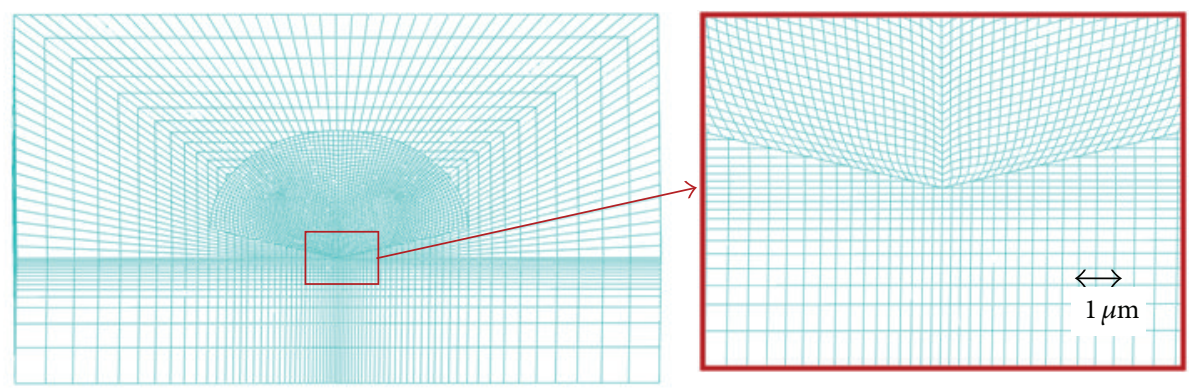

Figure 1: Finite element meshes of the numerical model of conical indentation (only half model is shown for better view). The image in the red box provides the details of the mesh in the region of contact.

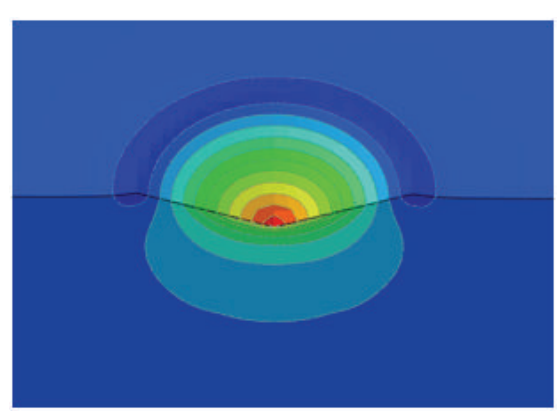

(a)

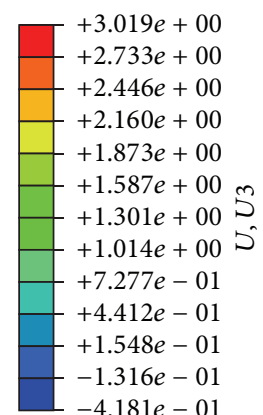

$-4.181 e-01$

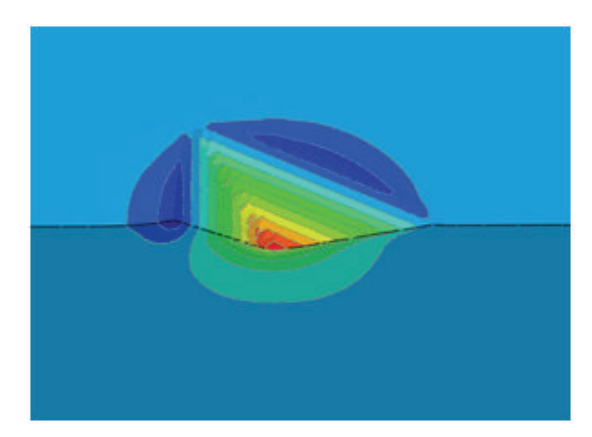

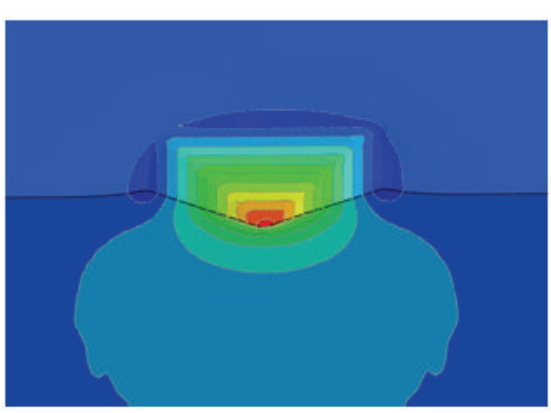

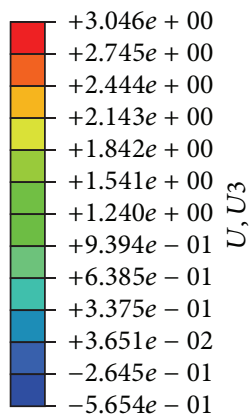

(b)

(c)

Figure 2: Displacement fields at indentation depth of $3 \mu \mathrm{m}$ for conical indenter (a), Vickers indenter (b), and Berkovich indenter (c). Only half of each model is shown. Note that the negative values of displacement (U3) indicate pileup of the material.

further increase of element number led to little change of the $P$ - $h$ curves.

Similar to experiments, the $P$ - $h$ curves are extracted directly from the calculated results of each simulation. Other properties such as hardness and elastic modulus are derived from the $P$ - $h$ curves following the procedure given in Section 1 of this paper. The errors related to FEA include modeling error, discretization error, and numerical error. Since all of the simulation models are identical in the sense of modeling, loading, meshing, contact condition, and solver and the only differences are the geometry of indenters and the properties of testing materials, the simulated results can accurately reflect the role of these different inputs in instrumented indentation. Actually, one advantage of the "numerical experiment" is that it excludes all other factors that may influence the results and focuses on one or two concerned terms, which is very hard to achieve in real experiments.

\section{Results and Discussion}

Figure 2 displays the displacement field in the loading direction at a penetration depth of $3 \mu \mathrm{m}$ for the three indentations. The current $Y$ and $E$ are $800 \mathrm{MPa}$ and $100 \mathrm{GPa}$, respectively. Only half model is shown for better view. Note from the legend that positive values of displacement (U3) represent penetration into the material, while negative values indicate pileup. Clearly, pileup exists in each of the situations, demonstrating that corrections are required when using traditional process equations (2) and (3). Moreover, although 


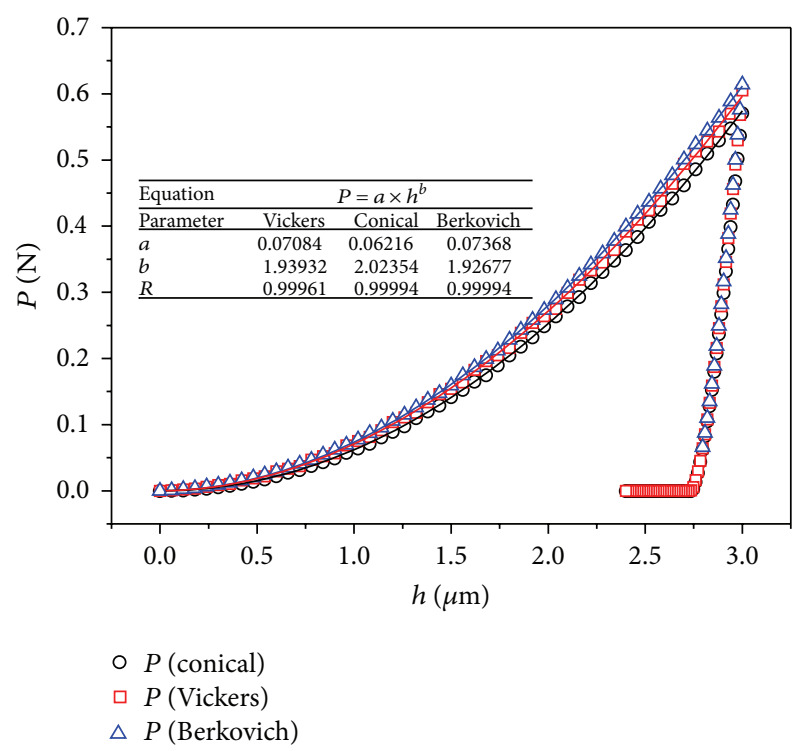

FIGURE 3: Force-displacement curves for different kinds of indenters. The solid lines are the power law fitting of the loading parts. $E=100 \mathrm{GPa}, Y=800 \mathrm{MPa}$.

the geometry functions of the three indenters are the same, their pileup patterns are rather different. These differences indicate that axial symmetric conical indenter may not be representative for the 3-side Berkovich indenter or 4-side Vickers indenter, especially when pileup plays a role.

The evolution of force $P$ with respect to indentation depth $h$ is plotted in Figure 3. The loading part of each indentation curve is fitted by the power law function. The fitting exponents, $b$, for all the simulations are between 1.93 and 2.02, corresponding well with the prediction by (12). The true contact area, $A_{c}$, between the indenter and the material is easy to obtain from the simulation. The true projected contact area, $A$, can be derived from $A_{c}$ through simple geometric relationships. Figure 4 displays the $A$ - $h$ curves for the three kinds of indentations. Although they behave differently in Figure 2, the three kinds of indenters present identical $A$ $h$ curves given the same $Y$ and $E$. The loading part of the $A$ - $h$ curves is also fitted by power law function and it gives the values of $b$ ranging from 2.0 to 2.1, in accordance with the prediction by (13). A reference area $A_{r}$, defined as the projected area calculated from indentation depth $h$ by (8) is given in Figure 4. Obviously, $A_{r}$ is significantly smaller than the true projected areas when the indentation depth is larger than $1 \mu \mathrm{m}$, signifying essential error may be introduced if the effect of pileup is ignored.

The dimensionless functions, that is, $\phi_{P L}=P /\left(E h^{2}\right)$, $\phi_{A}=A / h^{2}, \phi_{h f}=h_{f} / h_{\max }$, and $\phi_{S}=S /(E h)$, were plotted with respect to $Y / E$ in Figures 5, 6, 7, and 8, respectively. It is obvious from Figure 5 that these derived quantities lie in a single curve for each indenter. Thus $\phi_{P L}$ is a function of $Y / E$, as predicted by (12). Nevertheless, these functions are not exactly the same for each indenter. When $Y / E$ is larger than 0.01 (corresponding to materials like sodalime glass, high strength steels, titanium alloys, and so on),

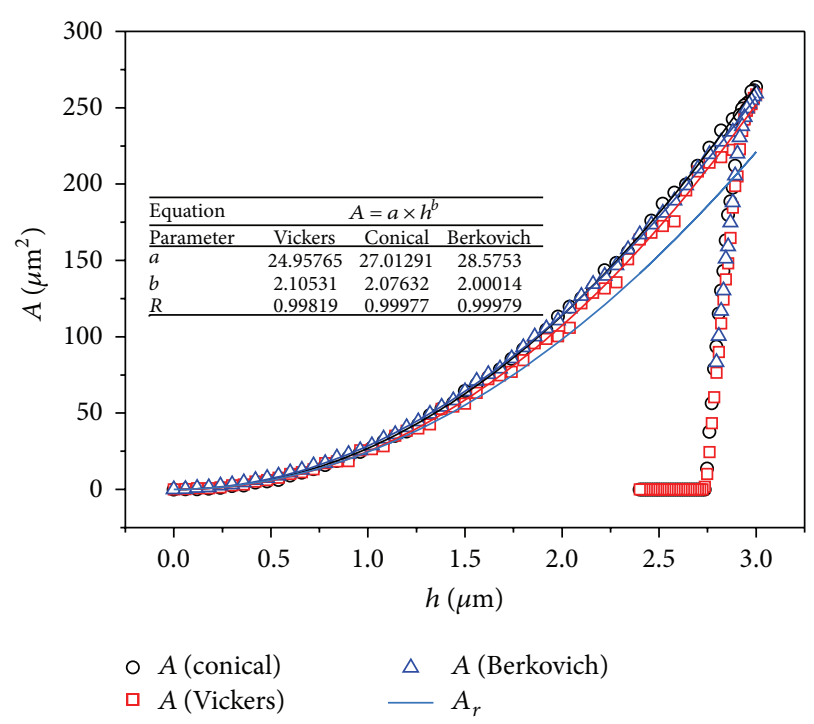

FIGURE 4: True projected area-displacement curves for three kinds of indenters. $A_{r}$ is the reference area that is calculated from the indentation depth by using the area function of the indenters. The solid lines are the power law fitting of the loading parts. $E=$ $100 \mathrm{GPa}, Y=800 \mathrm{MPa}$.

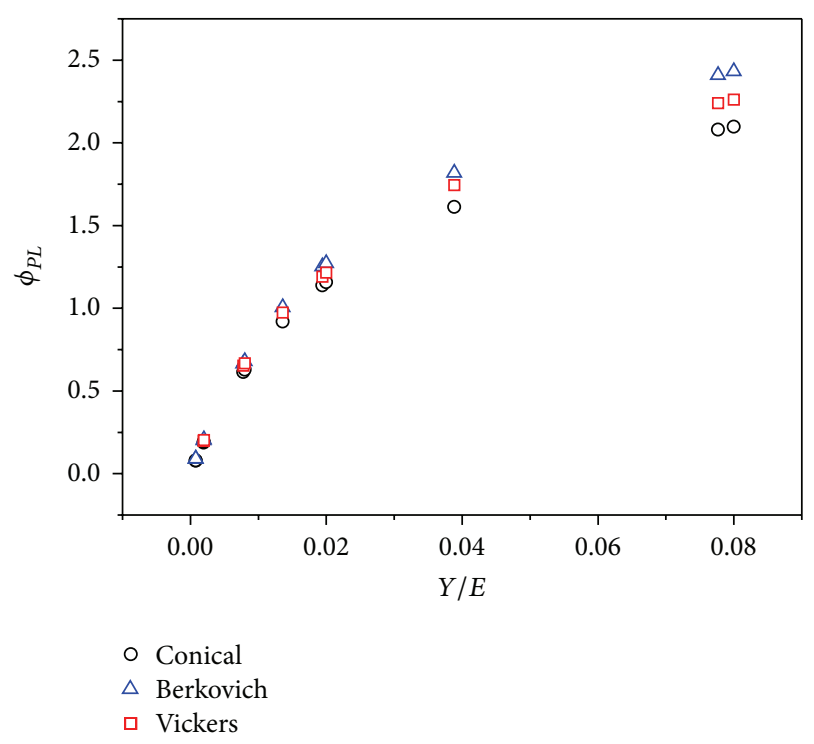

FIGURE 5: The variation of dimensionless function $\phi_{P L}=P /\left(E h^{2}\right)$ with respect to $Y / E$ for different kinds of indenters.

the differences among the data derived from the three indenters become evident. Similarly, the dimensionless function for projected contact area $\phi_{A}$ is a function of $Y / E$ and is different for each indenter. But, different from $\phi_{P L}$, the discrepancy of $\phi_{A}$ becomes significant when $Y / E$ is smaller than 0.02 (corresponding to materials like aluminum alloys, low carbon steels, and so on). The dimensionless function $\phi_{h f}$ decreases monotonously with increasing $Y / E$ and appears independent of the geometry of the indenter (see Figure 7 ). $h_{f} / h_{\max }$ is a very useful quantity, because it is easy to obtain from the $P$ - $h$ curve and many researchers $[7-9,14]$ took it as an indicator 


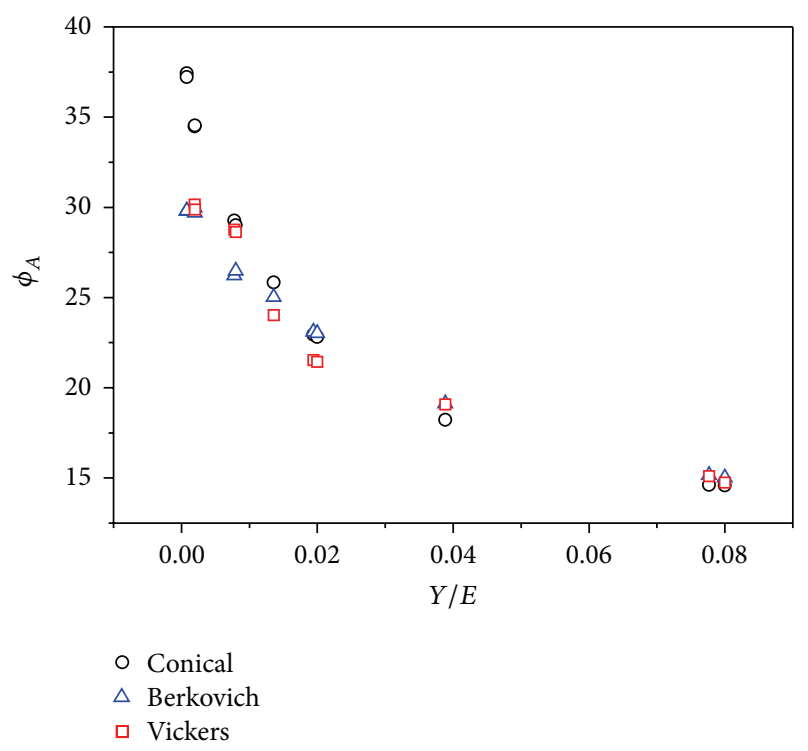

Figure 6: The variation of dimensionless function $\phi_{A}=A / h^{2}$ with respect to $Y / E$ for different kinds of indenters.

for pileup or sink-in. The dimensionless contact stiffness $\phi_{S}$ becomes smaller as $Y / E$ increases but the differences among different indenters become more significant. Since the contact stiffness is essential for the derivation of the elastic modulus (see (6)), its scattering among different indenters may lead to significant variation of the calculated elastic modulus.

The projected contact area $A$ is an important quantity that directly influences the measured hardness. The dependence of the normalized projected contact area $A / A_{r}$ on $h_{f} / h_{\max }$ is given in Figure 9. The true projected contact areas (solid symbols) and those calculated by the Oliver and Pharr $(\mathrm{O} / \mathrm{P})$ model [1] (hollow symbols) are all presented. The red dashed line, where $A / A_{r}$ is 1.0 , denotes no pileup or sinkin. All of the hollow symbols are below the dashed line, showing that the $\mathrm{O} / \mathrm{P}$ model considers sink-in only and could lead to significant error if $h_{f} / h_{\max }$ is larger than 0.8 . The solid symbols intersect with the dashed line at around $h_{f} / h_{\max }=0.85$ for all the three indenters, indicating that direct penetration depth and area function could be used to calculate projected contact area $A$ at this point (8). Our simulation results also reveal that less than $10 \%$ error will be introduced if pileup or sink-in effect is ignored when $0.8<$ $h_{f} / h_{\max }<0.9$ (corresponding $0.008<Y / E<0.02$ ) for Berkovich indentation. However, this error increases to more than $20 \%$ for conical or Vickers indentation. This observation demonstrates that Berkovich indenter is less sensitive to pileup or sink-in than Vickers and conical indenters within this region. If pileup and sink-in are ignored, the maximum error could be larger than $50 \%$ for the conical indenter.

The derived elastic modulus of the material depends on the contact stiffness $S$, the projected contact area $A$, and the correction factor $\beta(6)$. The differences of $S$ and $A$ could lead to variation of $E$ for different indenters. Figure 10 displays the dependence of calculated $E$ on $h_{f} / h_{\max }$.

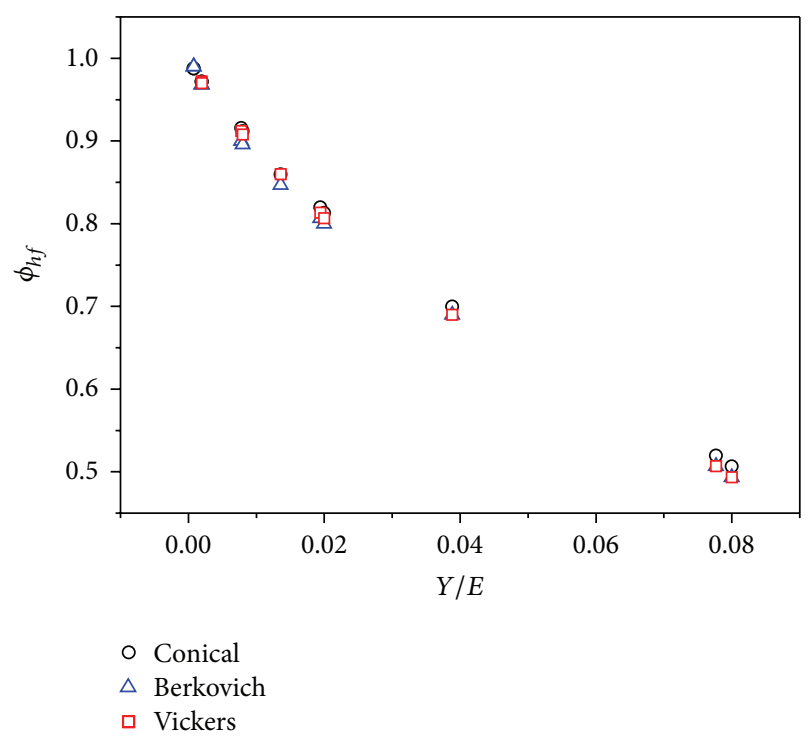

FIGURE 7: The variation of dimensionless function $\phi_{h f}=h_{f} / h_{\max }$ with respect to $Y / E$ for different kinds of indenters.

The solid symbols represent $E$ derived from the true projected contact area, while hollow symbols represent $E$ derived from the O/P model. Obviously, all of the values of calculated $E$ are larger than the input value and the $\mathrm{O} / \mathrm{P}$ method could introduce more error if improper correction factor $\beta$ is used. Figure 10 shows that the error of calculated $E$ increases with increment of $h_{f} / h_{\max }$, demonstrating that the correction factor $\beta$ should not be a constant but a function of $h_{f} / h_{\max }$; that is, $\beta=a+b\left(h_{f} / h_{\max }\right)$. For the non-work-hardening materials in this work, $a$ and $b$ are 1.021 and $0.358,0.832$ and 0.526 , and 0.759 and 0.512 for the Berkovich, Vickers, and conical indenters, respectively. Another noteworthy feature in Figure 10 is that Berkovich indentation will yield higher elastic modulus than Vickers and conical indentations, indicating again that replacing Berkovich and Vickers by conical indenter is not appropriate. An approximate relationship of $E$ among different indenters can be given as $E_{\text {Berkovich }}=$ $1.06 E_{\text {Vickers }}=1.13 E_{\text {Conical }}$. This estimation leads to less than $5 \%$ error when $h_{f} / h_{\max }<0.95$ (corresponding to $Y / E>$ 0.002).

Figure 11 illustrates the variation of the normalized hardness $H_{\mathrm{O} / \mathrm{P}} / H_{\text {true }}$ with respect to $h_{f} / h_{\max } \cdot H_{\mathrm{O} / \mathrm{P}}$ and $H_{\text {true }}$ are derived by using the $\mathrm{O} / \mathrm{P}$ model and the true projected contact area, respectively. The $\mathrm{O} / \mathrm{P}$ hardness, as expected, is larger than the true hardness for all cases. When $h_{f} / h_{\max }<$ $0.8, H_{\mathrm{O} / \mathrm{P}} / H_{\text {true }} \approx 1.1$ and this approximation will introduce no more than $5 \%$ error for all the three indenters. However, this ratio increases sharply when $h_{f} / h_{\max }>0.8$. For conical indentation, $H_{\mathrm{O} / \mathrm{P}} / H_{\text {true }}$ rises monotonously up to 1.54 , but the maximum values for Berkovich and Vickers indentation are less than 1.3. Thus, conical indentation fails again to represent Berkovich and Vickers indentations.

The hardness is normalized by yield stress of the material and plotted against $h_{f} / h_{\max }$ in Figure 12. In the case that $H$ is calculated by the $\mathrm{O} / \mathrm{P}$ method, similar trends of $H / Y$ are 


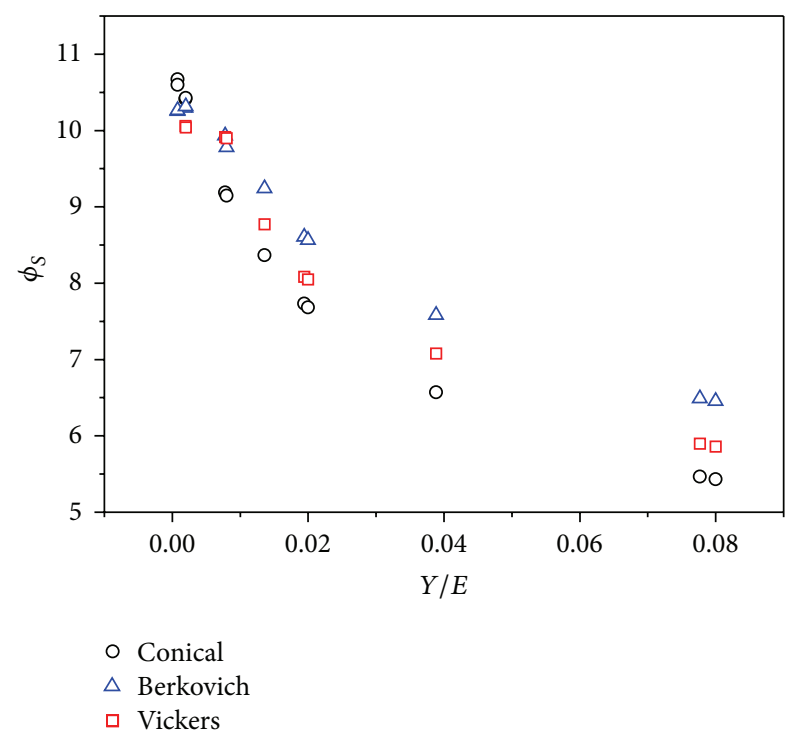

FIGURE 8: The variation of dimensionless function $\phi_{S}=S /(E h)$ with respect to $Y / E$ for different kinds of indenters.

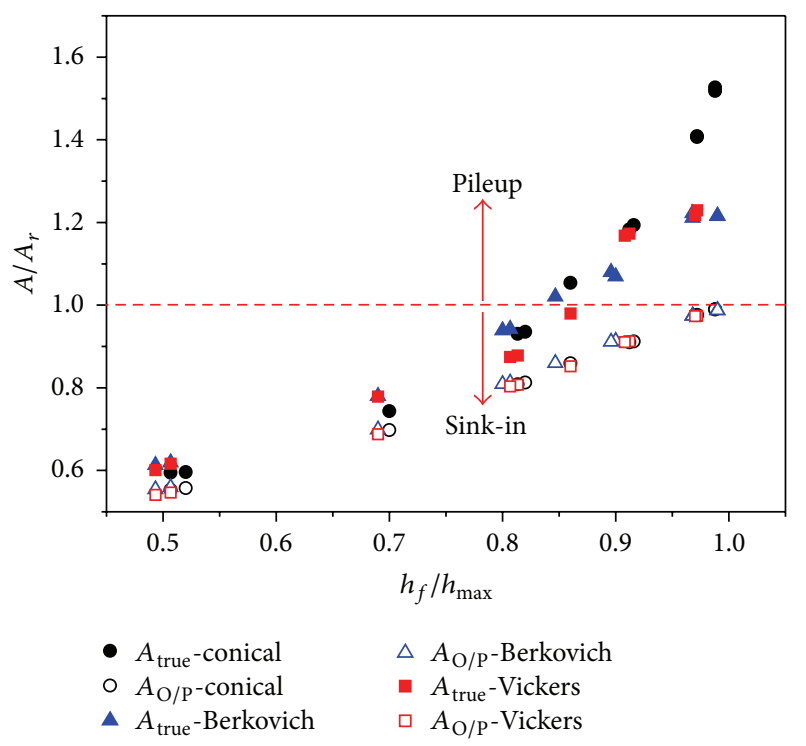

FIgURE 9: Influence of $h_{f} / h_{\max }$ on the projected contact area. The projected contact area is normalized by $A_{r}$, the area calculated by the indenter geometry function. The solid icons stand for the true projected contact area derived from finite element models, while the hollow icons represent the projected contact area calculated by Oliver and Pharr model [1].

observed for all the three indenters in Figure 12, although there are still differences in the magnitudes. When $h_{f} / h_{\max }$ approaches unity, the maximum values of $H / Y$ are achieved and are larger than 4.2 for all indenters. When true hardness is applied, different patterns of $H / Y$ are found for different indenters. The relationship between hardness and yield stress, $C=H / Y$, was firstly proposed by Tabor [27], where $C$ is the constraint factor. Tabor analyzed the rigid/plastic deformation of Vickers indentation and claimed that the hardness of metals is about 2.9 3.0 times its flow stress at $8 \sim 10 \%$ strain; that is, $C \approx 2.9 \sim 3.0$. Since rigid/plastic deformation corresponds to $h_{f} / h_{\max }=1$, our numerical results could be compared with $C$. Our simulation shows that $H / Y \approx 3.3$ for Vickers indentation when $h_{f} / h_{\max }$ approaches unity, slightly larger than Tabor's value. Different from Vickers and Berkovich, the value of $H / Y$ for conical indentation plateaus at about 2.7, which is close to that calculated by Bolshakov and Pharr [7]. Clearly, $C$ is not a constant but varies with $h_{f} / h_{\max }$. The value of $C$ also depends on the indenter geometry. For soft material, that is, $h_{f} / h_{\max }$ close to 1 (or $Y / E$ close to zero from Figure 7), $C$ reaches 2.7, 3.3, and 3.6 for conical, Vickers, and Berkovich indentation, respectively. However, $C$ might be lower than 2.0 for some hard materials.

It should be noted that $h_{f} / h_{\max }=0.8($ or $Y / E=0.02)$ is a critical point, below which the true projected contact area, hardness, and Tabor factor are almost proportional to the values derived from Oliver and Pharr model. In that case, the results by traditional method could still be used if a correction factor is applied (hardness divided by, e.g., 1.1). However, this factor could not be used if $h_{f} / h_{\max }>0.8$ (or $Y / E<0.02$ ), where the relation between true values and Oliver-Pharr results becomes different for different indenters. As $Y / E$ is less than 0.02 for most of the engineering materials, the corrections should be more careful.

Our experimental results [15] on niobium could be used to compare with some of the simulation results. The commercially pure niobium disks with diameter of $10.0 \mathrm{~mm}$ and thickness of $1.0 \mathrm{~mm}$ were processed by high pressure torsion (HPT) at room temperature. The Von Mises equivalent strain at the edge of the disk reaches about 90 , while it is theoretically zero at the disk center. The large deformation by HPT substantially enhances the yield strength of the material while reducing its ductility. One distinct characteristic of HPT-ed materials is their vanished work-hardening ability, which is in keeping with the assumption of elastic-perfectplastic material in our simulation. In fact, the material undergoes deformation even at the center of the disk during HPT. The Berkovich hardness is $1.2 \mathrm{GPa}$ at the niobium disk center [15], corresponding to about $400 \mathrm{MPa}$ for yield stress $Y$ from Figure 12. Considering that the strength of as received niobium is less than $200 \mathrm{MPa}$, the enhancement in strength and at the same time reduction in ductility at the disk center would be significant. Therefore, assuming elasticperfect-plastic material property at the disk center will not lead to essential error. The HPT process does not change the elastic modulus remarkably. Thus, the value of $Y / E$ is larger at the edge than at the center of the disk. Figure 13 presents the indentation geometry at the center (a) and edge (b) of the HPT processed niobium disk. It can be observed that significant pileup appears at the center (a2), while neither sink-in nor pileup is observed at the edge (b2). The values of $h_{f} / h_{\max }$ are 0.93 and 0.81 for Figures 13(a) and 13(b), respectively. The corresponding values of $Y / E$ are estimated to be 0.0057 and 0.019 from Figure 7 . This experimental observation of pileup or sink-in is well predicted by Figure 9 . 


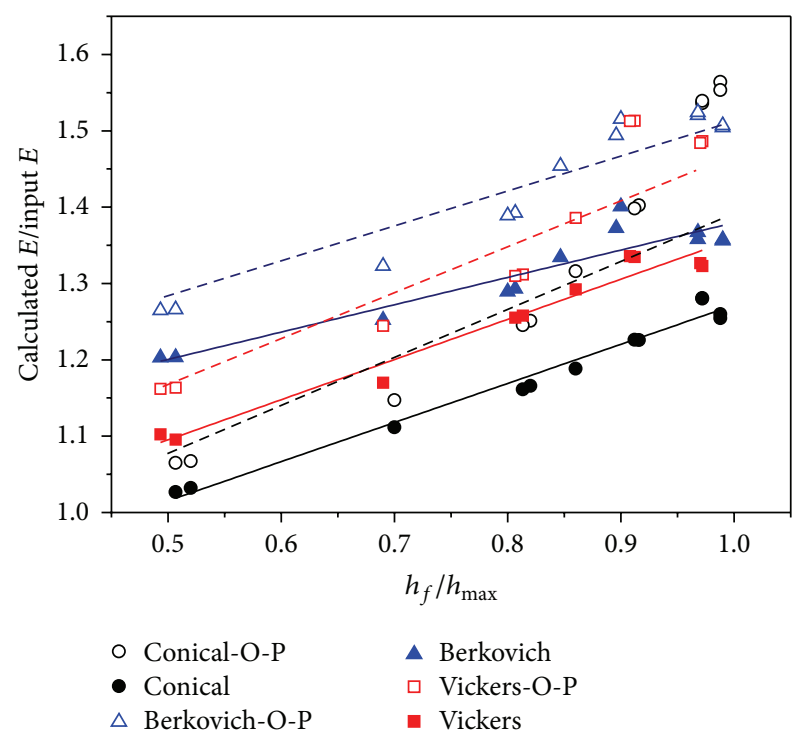

FIGURE 10: Influence of $h_{f} / h_{\max }$ on the calculated elastic modulus. The modulus is normalized by the input $E$ of the numerical model. The solid icons stand for the elastic modulus derived from true projected contact areas (6), while the hollow icons represent the modulus derived by Oliver and Pharr model [1]. The solid and dashed lines are linear fitting of solid icons and hollow icons, respectively.

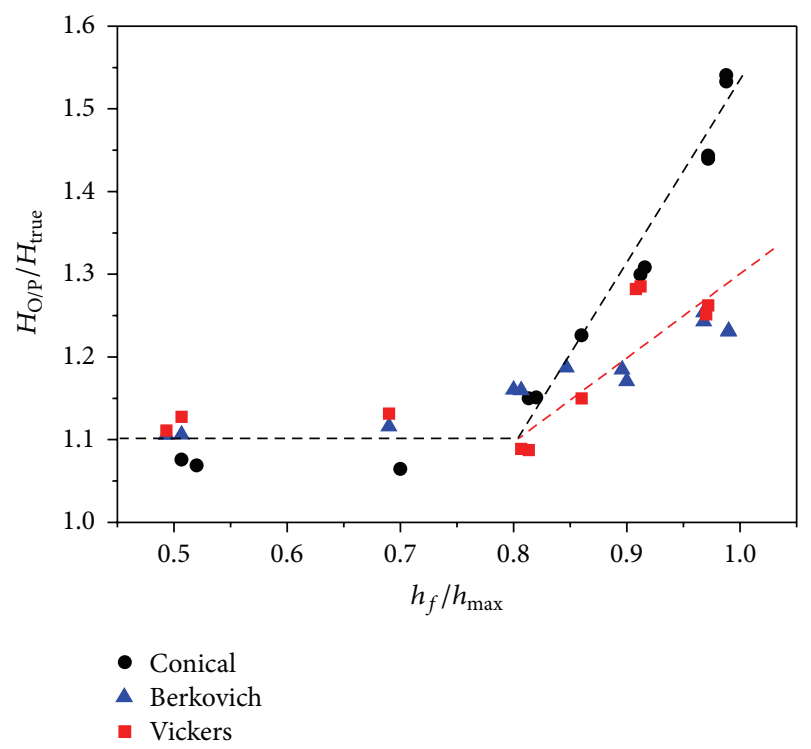

FIgURE 11: Effect of $h_{f} / h_{\max }$ on $H_{\mathrm{O} / \mathrm{P}} / H_{\text {true }}$, the ratio of hardness derived by Oliver and Pharr model to that calculated by using true projected contact area.

\section{Summary and Conclusion}

Three kinds of indentation, namely, conical, Berkovich, and Vickers, were comparatively studied by means of numerical simulations. The effect of pileup on the derived quantities was carefully examined. Dimensionless analysis was adopted to

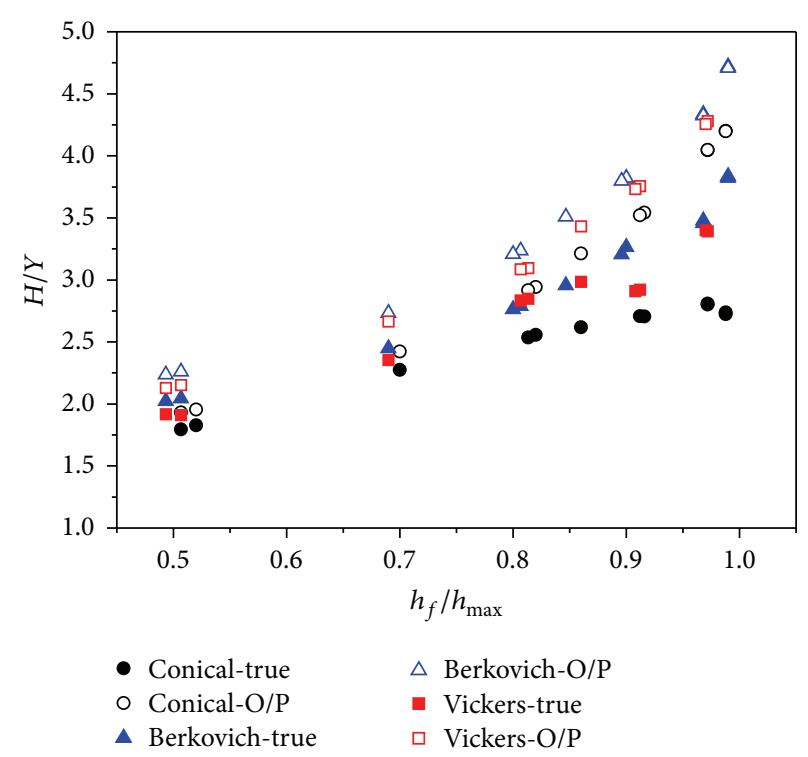

Figure 12: Influence of $h_{f} / h_{\max }$ on $H / Y$. The solid icons stand for the hardness derived from true projected contact areas, while the hollow icons represent those derived by Oliver and Pharr model [1].

acquire the principal governing parameters of the process. The main findings are summarized in the following:

(1) The dimensionless functions $\phi_{P L}, \phi_{A}, \phi_{h f}$, and $\phi_{S}$, which represent loading, projected contact area, final indentation depth, and contact stiffness, respectively, are all functions of $Y / E$ only for the elastic-perfectplastic material considered in this work. However, these functions are not the same for different kinds of indenters.

(2) When $h_{f} / h_{\max }>0.85$, the effect of pileup becomes significant. Failure to account for the effect of pileup may lead to more than $50 \%$ error in calculating elastic modulus and hardness.

(3) The derived elastic modulus could be different if different indenters are adopted, even if the effect of pileup is considered. An approximate relationship of $E$ of the sample measured using different indenters can be given by $E_{\text {Berkovich }}=1.06 E_{\text {Vickers }}=$ $1.13 E_{\text {Conical }}$. This estimation leads to less than $5 \%$ error when $h_{f} / h_{\max }<0.95$ (corresponding to $Y / E>$ 0.002).

(4) The Tabor factor, $C=H / Y$, is not a constant but varies with $h_{f} / h_{\max }$. For soft material, that is, $h_{f} / h_{\max }$ close to unity (or $Y / E$ close to zero from Figure 7), $C$ reaches 2.7, 3.3, and 3.6 for conical, Vickers, and Berkovich indentation, respectively.

The above conclusions are acquired based on a group of specific materials whose strain hardening is ignored and the effect of Poisson's ratio is not considered. Moreover, the timedependent (or strain-rate dependent) characteristics of the indentation process for some materials are not reckoned in for present work. Therefore, application of these results 


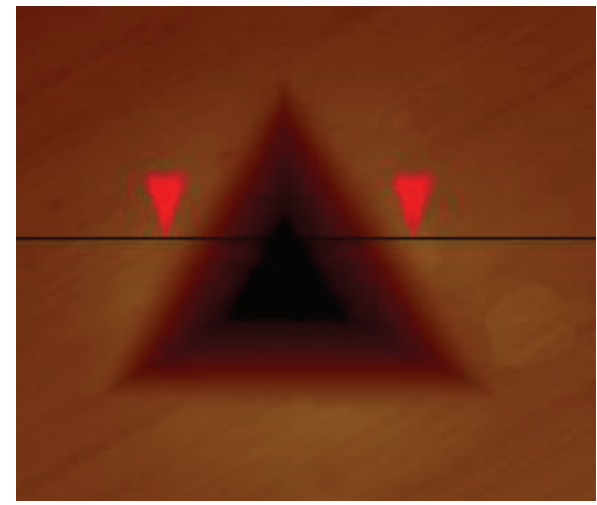

(a1)

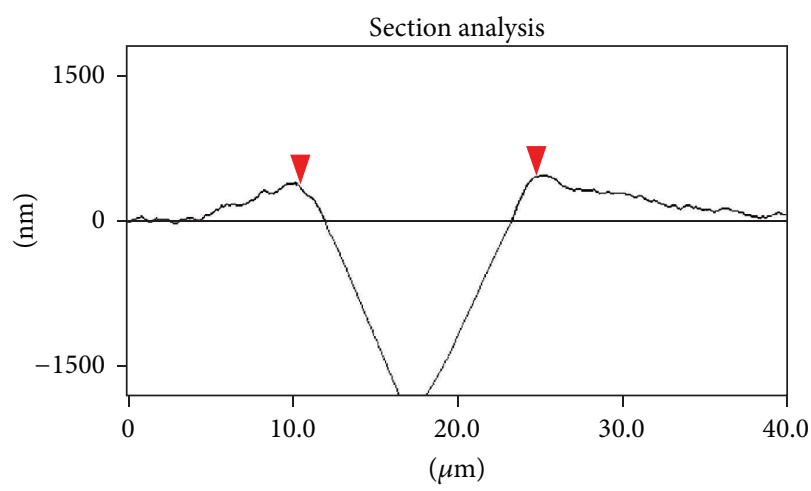

(a2)

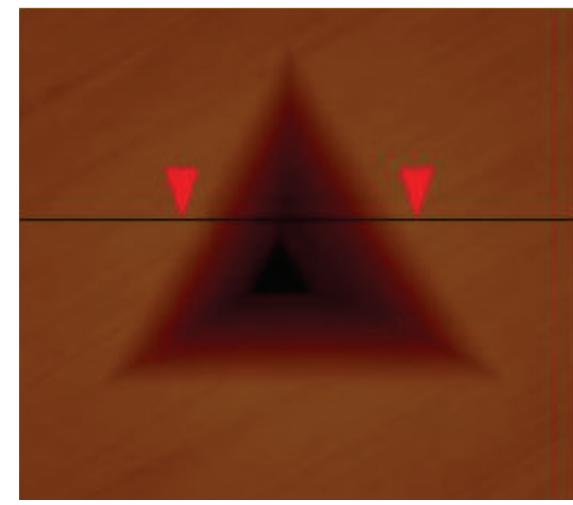

(b1)

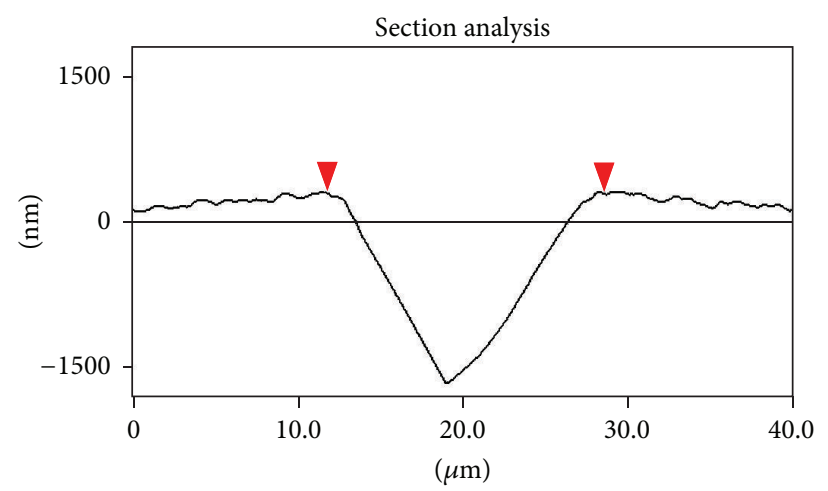

(b2)

(b)

Figure 13: Appearance of the indentation geometry by AFM at the center (a) and edge (b) of the HPTed niobium disk. It can be observed that, at the center of the disk, significant pileup effect is present (a2), while, at the edge, neither sink-in nor pileup effect is observed (b2) [15].

should be conditional. The role of strain hardening and strain rate hardening might be significant in determining the properties of the testing materials and should be further investigated.

\section{Conflict of Interests}

The authors declare that there is no conflict of interests regarding the publication of this paper.

\section{Acknowledgments}

The authors would like to thank the financial support by National Natural Science Foundation of China (nos. 11102168, 11102166, 11472227, 11202168, and 10932008), the 111 Project (no. B07050), and the Fundamental Research Funds for the Central Universities (no. 310201401JCQ01001).

\section{References}

[1] W. C. Oliver and G. M. Pharr, "Improved technique for determining hardness and elastic modulus using load and displacement sensing indentation experiments," Journal of Materials Research, vol. 7, no. 6, pp. 1564-1580, 1992.
[2] Y.-T. Cheng and C.-M. Cheng, "Scaling, dimensional analysis, and indentation measurements," Materials Science and Engineering: R: Reports, vol. 44, no. 4-5, pp. 91-149, 2004.

[3] L. Cheng, X. Xia, L. E. Scriven, and W. W. Gerberich, "Sphericaltip indentation of viscoelastic material," Mechanics of Materials, vol. 37, no. 1, pp. 213-226, 2005.

[4] W. C. Oliver and G. M. Pharr, "Measurement of hardness and elastic modulus by instrumented indentation: advances in understanding and refinements to methodology," Journal of Materials Research, vol. 19, no. 1, pp. 3-20, 2004.

[5] Y. Z. Guo, Y. L. Li, X. L. Wu, and Q. Wei, "A modified criterion for shear band formation in bulk metallic glass under complex stress states," Materials Science and Engineering A, vol. 527, no. 10-11, pp. 2613-2620, 2010.

[6] B. Storåkers, S. Biwa, and P.-L. Larsson, "Similarity analysis of inelastic contact," International Journal of Solids and Structures, vol. 34, no. 24, pp. 3061-3083, 1997.

[7] A. Bolshakov and G. M. Pharr, "Influences of pileup on the measurement of mechanical properties by load and depth sensing indentation techniques," Journal of Materials Research, vol. 13, no. 4, pp. 1049-1058, 1998.

[8] Y.-T. Cheng and C.-M. Cheng, "Relationships between hardness, elastic modulus, and the work of indentation," Applied Physics Letters, vol. 73, no. 5, pp. 614-616, 1998. 
[9] Y.-T. Cheng and C.-M. Cheng, "Scaling approach to conical indentation in elastic-plastic solids with work hardening," Journal of Applied Physics, vol. 84, no. 3, pp. 1284-1291, 1998.

[10] Y.-T. Cheng and C.-M. Cheng, "Scaling relationships in conical indentation of elastic-perfectly plastic solids," International Journal of Solids and Structures, vol. 36, no. 8, pp. 1231-1243, 1999.

[11] Y.-T. Cheng, Z. Y. Li, and C.-M. Cheng, "Scaling relationships for indentation measurements," Philosophical Magazine A: Physics of Condensed Matter, Structure, Defects and Mechanical Properties, vol. 82, no. 10, pp. 1821-1829, 2002.

[12] M. Dao, N. Chollacoop, K. J. van Vliet, T. A. Venkatesh, and S. Suresh, "Computational modeling of the forward and reverse problems in instrumented sharp indentation," Acta Materialia, vol. 49, no. 19, pp. 3899-3918, 2001.

[13] A. E. Giannakopoulos, P. L. Larsson, and R. Vestergaard, "Analysis of Vickers indentation," International Journal of Solids and Structures, vol. 31, no. 19, pp. 2679-2708, 1994.

[14] P.-L. Larsson, A. E. Giannakopoulos, E. Söderlund, D. J. Rowcliffe, and R. Vestergaard, "Analysis of Berkovich indentation," International Journal of Solids and Structures, vol. 33, no. 2, pp. 221-248, 1996.

[15] Y. Z. Guo, N. A. Behm, J. P. Ligda et al., "Critical issues related to instrumented indentation on non-uniform materials: application to niobium subjected to high pressure torsion," Materials Science and Engineering A, vol. 586, pp. 149-159, 2013.

[16] N. Huber, W. D. Nix, and H. Gao, "Identification of elasticplastic material parameters from pyramidal indentation of thin films," Proceedings of the Royal Society of London A: Mathematical, Physical and Engineering Science, vol. 458, no. 2023, pp. 1593-1620, 2002.

[17] N. Huber and C. Tsakmakis, "Determination of constitutive properties from spherical indentation data using neural networks. Part II: plasticity with nonlinear isotropic and kinematic hardening," Journal of the Mechanics and Physics of Solids, vol. 47, no. 7, pp. 1589-1607, 1999.

[18] N. Huber and C. Tsakmakis, "Determination of constitutive properties from spherical indentation data using neural networks. Part I: the case of pure kinematic hardening in plasticity laws," Journal of the Mechanics and Physics of Solids, vol. 47, no. 7, pp. 1569-1588, 1999.

[19] T. Y. Tsui and G. M. Pharr, "Substrate effects on nanoindentation mechanical property measurement of soft films on hard substrates," Journal of Materials Research, vol. 14, no. 1, pp. 292-301, 1999.

[20] T. Y. Tsui, J. Vlassak, and W. D. Nix, "Indentation plastic displacement field: part I. The case of soft films on hard substrates," Journal of Materials Research, vol. 14, no. 6, pp. 21962203, 1999.

[21] T. Y. Tsui, J. Vlassak, and W. D. Nix, "Indentation plastic displacement field: part II. The case of hard films on soft substrates," Journal of Materials Research, vol. 14, no. 6, pp. 2204-2209, 1999.

[22] N. Huber and J. Heerens, "On the effect of a general residual stress state on indentation and hardness testing," Acta Materialia, vol. 56, no. 20, pp. 6205-6213, 2008.

[23] T. Y. Tsui, W. C. Oliver, and G. M. Pharr, "Influences of stress on the measurement of mechanical properties using nanoindentation: part I. Experimental studies in an aluminum alloy," Journal of Materials Research, vol. 11, no. 3, pp. 752-759, 1996.
[24] S. Carlsson and P.-L. Larsson, "On the determination of residual stress and strain fields by sharp indentation testing. Part I: theoretical and numerical analysis," Acta Materialia, vol. 49, no. 12, pp. 2179-2191, 2001.

[25] S. Carlsson and P.-L. Larsson, "On the determination of residual stress and strain fields by sharp indentation testing. Part II: experimental investigation," Acta Materialia, vol. 49, no. 12, pp. 2193-2203, 2001.

[26] L. Brand, "The Pi theorem of dimensional analysis," Archive for Rational Mechanics and Analysis, vol. 1, pp. 35-45, 1957.

[27] D. Tabor, The Hardness of Metals, Oxford University Press, London, UK, 2000. 

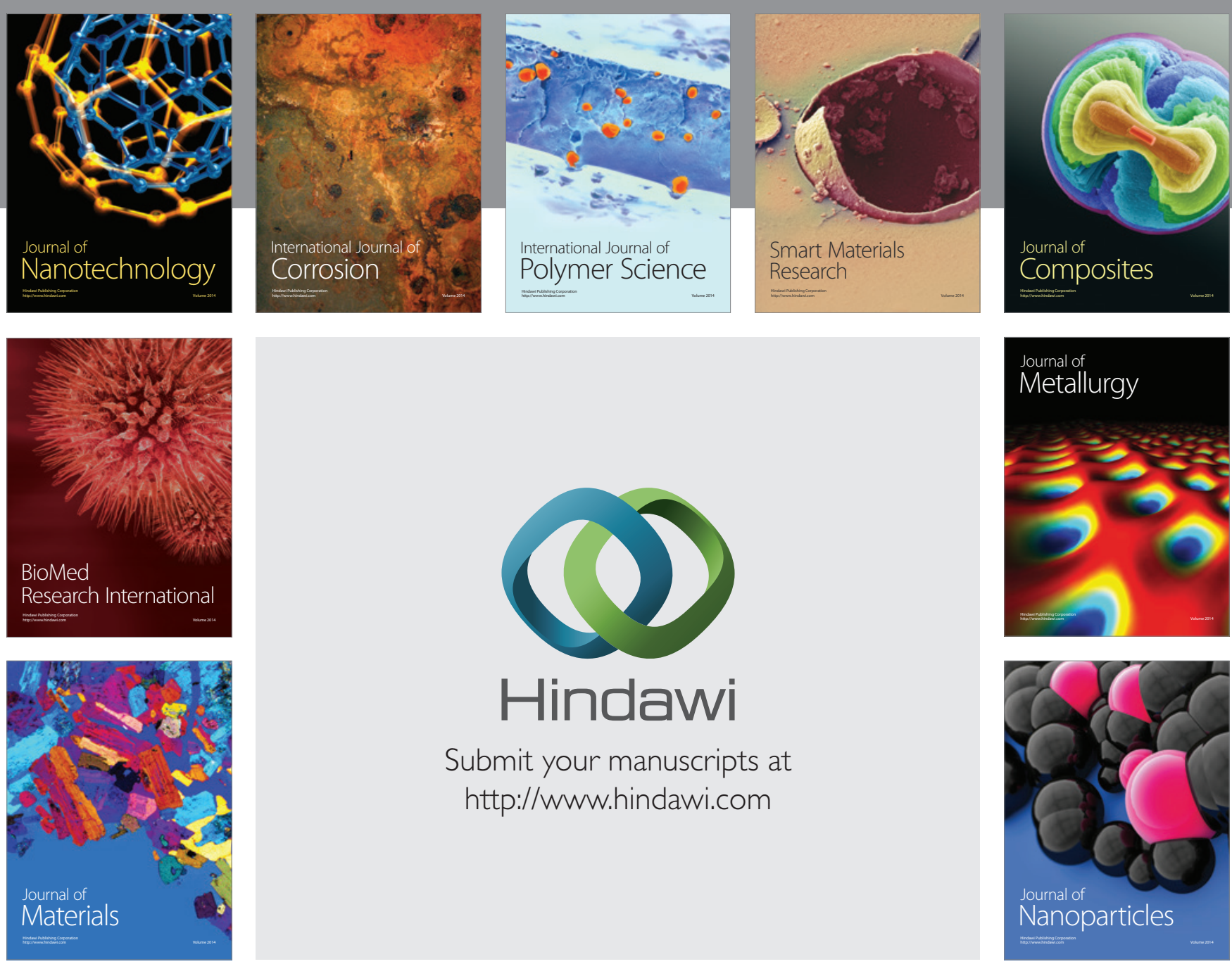

Submit your manuscripts at http://www.hindawi.com
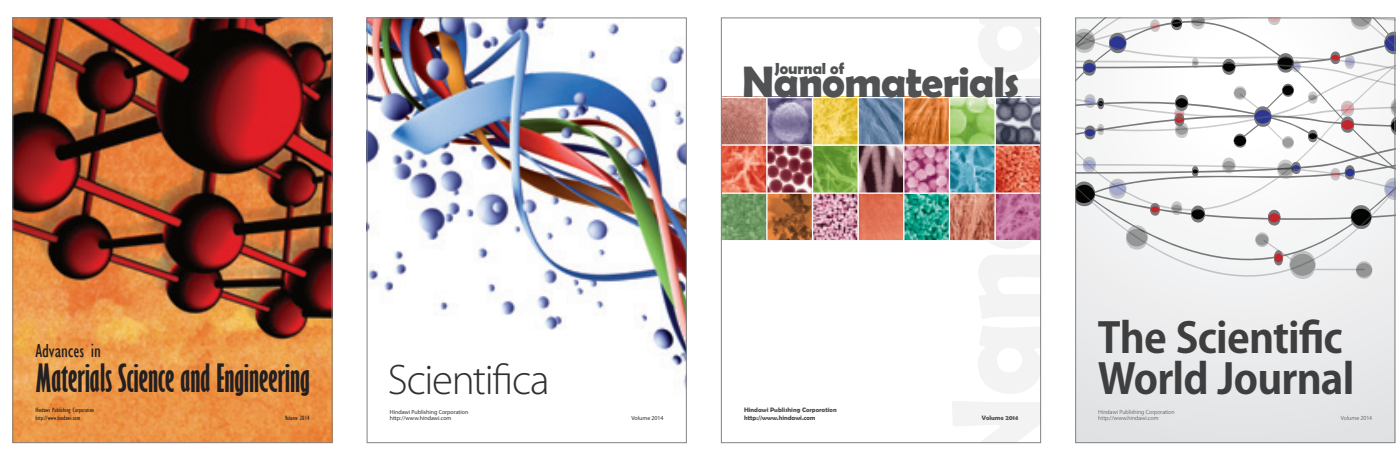

\section{The Scientific World Journal}
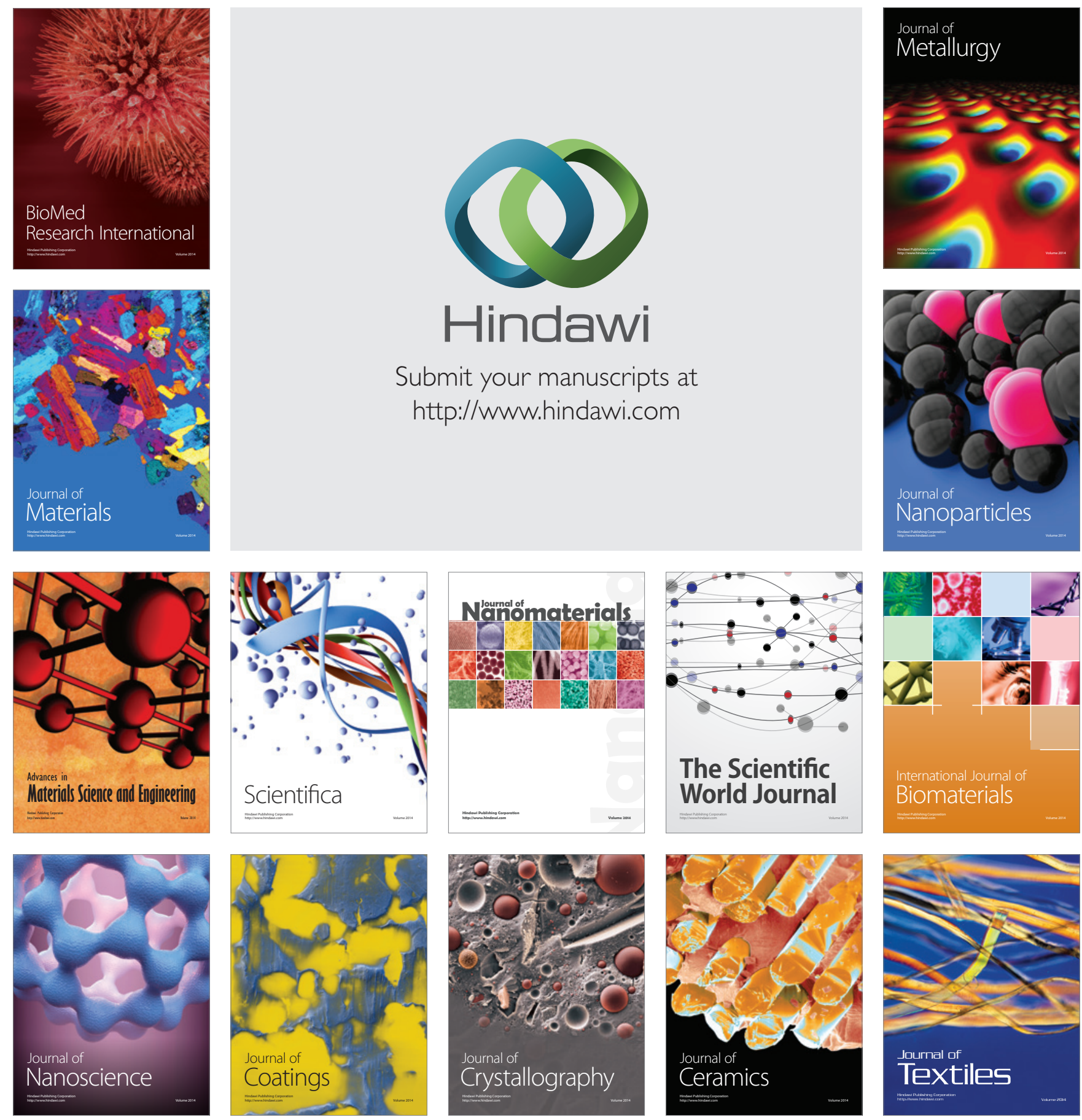